\title{
THE ADDITIVE INVERSE EIGENVALUE PROBLEM AND TOPOLOGICAL DEGREE
}

\author{
J. C. ALEXANDER ${ }^{1}$
}

\begin{abstract}
A short proof using topological degree is given of the additive inverse eigenvalue problem: The diagonal elements of any square complex matrix can be altered so as to cause the altered matrix to have any prescribed set of eigenvalues.
\end{abstract}

The (finite-dimensional) additive inverse eigenvalue problem is the following. Given an $n$ by $n$ complex matrix $M$ and a set of $n$ complex numbers $\xi_{1}, \ldots, \xi_{n}$, possibly with repetitions, does there exist a diagonal matrix $D$ such that the eigenvalues of $M+D$ are precisely $\xi_{1}, \ldots, \xi_{n}$ ?

The answer is yes. In fact generically in $\xi_{1}, \ldots, \xi_{n}$, there exist $n ! \operatorname{such} D$, and hence there are always between 1 and $n$ ! solutions $D$. This problem was solved by S. Friedland after some others had obtained various partial solutions. He has given two proofs. The first [2] is completely algebraic and the second [4] uses some powerful results from algebraic geometry. The purpose of this note is to give a short proof using topological degree. Degree theory is a standard tool for many analysts and the present proof may feel closer to home. Also, the fact that the degree is nonzero allows the use of methods developed by Kellogg, Li, and Yorke [5], [6] and Hirsch and Smale (unpublished) to find the solutions numerically. See [1, Theorem 4.2]. It should also be mentioned that Friedland has solved the multiplicative inverse eigenvalue problem (where one tries to specify the eigenvalues of $M D$ instead of $M+D$ ), also using degree theory [3]. An elegant general reference for degree theory is John Milnor's book [7].

We turn to the proof. Let $t^{n}+a_{1} t^{n-1}+\cdots+a_{n}$ be the monic polynomial with roots $\xi_{1}, \ldots, \xi_{n}$. We want the characteristic polynomial of $M+D$ to be $p$. Consider $f_{M}: \mathbf{C}^{n} \rightarrow \mathbf{C}^{n}$ defined by

$$
f_{M}(D)=\left(\alpha_{1}, \ldots, \alpha_{n}\right)
$$

if

$$
\operatorname{det}(t I-(M+D))=t^{n}+\alpha_{1} t^{n-1}+\cdots+\alpha_{n} .
$$

If $M=0$, the zero matrix, and $D$ has entries $d_{1}, d_{2}, \ldots, d_{n}$, then

Received by the editors October 15, 1976.

AMS (MOS) subject classifications (1970). Primary 15A18; Secondary 15-04.

${ }^{1}$ Partially supported by an NSF contract and a University of Maryland Faculty Research Grant. 


$$
f_{M}(D)=\left(t-d_{1}\right)\left(t-d_{2}\right) \cdots\left(t-d_{n}\right) .
$$

It can be checked that each $f_{M}$ is proper, that all $f_{M}$ are properly homotopic to $f_{0}$ and that $f_{0}$ has degree $n !$. Thus $f_{M}$ also has degree $n !$. These details can be checked, but we have chosen to proceed slightly differently by compactifying the problem and using degree defined for closed compact manifolds rather than work with proper maps.

Let complex projective space

$$
\mathbf{C} P^{n}=\left\{\left.\left[\alpha_{0}, \ldots, \alpha_{n}\right]\left|\alpha_{i} \in \mathbf{C}, \sum\right| \alpha_{i}\right|^{2} \neq 0\right\},
$$

where $\left[\alpha_{0}, \ldots, \alpha_{n}\right]=\left[\lambda \alpha_{0}, \ldots, \lambda \alpha_{n}\right]$ for any nonzero $\lambda \in \mathbf{C}$. We identify $C P^{n}$ with the space of all nonzero complex polynomials $\alpha_{0} t^{n}+\alpha_{1} t^{n-1}$ $+\cdots+\alpha_{n}$ modulo scalar multiples. In particular we consider $p \in \mathbf{C} P^{n}$. Let $D$ have entries $d_{1}, \ldots, d_{n}$. One-point compactify each copy of $\mathbf{C}$ to a Riemann sphere and consider $d_{i} \in \mathbf{C} \cup\{\infty\}=S^{2}$. We claim the map $f_{M}$ extends to a map

$$
\bar{f}_{M}: \underbrace{S^{2} \times \cdots \times S^{2}}_{n \text { times }} \rightarrow \mathbf{C} P^{n}
$$

To see that $f_{M}$ extends, let $M=\left(m_{i j}\right)$, and suppose $d_{1}$ is large. Then

$$
f_{M}(D)=\operatorname{det}\left(\begin{array}{cccc}
\frac{t}{d_{1}}-\frac{m_{11}}{d_{1}}-1 & \frac{m_{12}}{d_{1}} & \cdots & \frac{m_{1 n}}{d_{1}} \\
m_{21} & t-m_{22}-d_{2} & \cdots & m_{2 n} \\
\vdots & \vdots & & \vdots \\
m_{n 1} & m_{n 2} & \cdots & t-m_{n n}-d_{n}
\end{array}\right)
$$

$\in \mathbf{C} P^{n}$.

Thus if $d_{1}$ (or more generally if any subset of the $d$ 's) goes to infinity, the value of $f_{M}(D)$ approaches a limit in $\mathbf{C} P^{n}$. Note that $\bar{f}_{M}$ is complex analytic in both $M$ and $D$. Note also that if $k$ of the $d_{i}$ are equal to infinity, then the degree of the polynomial $\bar{f}_{M}(D)$ is $n-k$. In particular, $d_{i} \neq \infty$ for all $i$ if degree $f_{M}(D)=n$.

If $M=0$, the topological degree of $f_{M}$ is well known to be $n !$. (Two sketch proofs: (i) Treating $S^{2}=\mathrm{C} P^{1}$ as nonzero linear polynomials, $f_{0}:\left(S^{2}\right)^{n} \rightarrow$ $\mathbf{C} P^{n}$ is multiplication of polynomials. For all factors fixed at $0 \cdot t+1$ except for one, say $S_{i}^{2}$, the map $f_{0}$ is the inclusion of $S_{i}^{2}=\mathbf{C} P^{1}$ in $\mathbf{C} P^{n}$. Hence the generator of $H^{2}\left(\mathbf{C} P^{n}\right)$ maps to the generator of $H^{2}\left(S_{i}^{2}\right)$. Using the cup product structures of $H^{*}\left(\mathbf{C} P^{n}\right)$ and $H^{*}\left(\left(S^{2}\right)^{n}\right)$, we see $H^{2 n}\left(\mathbf{C} P^{n}\right) \rightarrow$ $H^{2 n}\left(\left(S^{2}\right)^{n}\right)$ is multiplication by $n$ !. (ii) Again treating $f_{0}:\left(S^{2}\right)^{n} \rightarrow \mathbf{C} P^{n}$ as multiplication of polynomials, it can be checked that a polynomial $q(t) \in$ $\mathbf{C} P^{n}$ is a regular value of $p_{0}$ if and only if all its roots $\left(r_{1}, \ldots, r_{n}\right)$ are distinct. The preimage of $q(t)$ is the $n$ ! products

$$
\pi=\left(t-r_{i_{1}}\right) \ldots\left(t-r_{i_{n}}\right)
$$


where $\left(i_{1}, \ldots, i_{n}\right)$ is a permutation of $(1, \ldots, n)$. The local degree at each $\pi$ is 1 , so the global degree is $n !$.) The map $f_{M}$ is homotopic to $f_{0}$, so the degree of $f_{M}$ is also $n$ !. In particular, $f_{M}$ is surjective and so there exists $D$ such that $f_{M}(D)=p$.

Moreover, by Sard's Theorem a generic set of $q \in \mathbf{C} P^{n}$ are regular values of $f_{M}$. The local degree of $f_{M}$ at each point in the inverse image of a regular $q$ is +1 since $f_{M}$ is complex analytic. Therefore $f_{M}^{-1}(q)$ has $n$ ! points if $q$ is regular. This completes the proof.

\section{REFERENCES}

1. S.-N. Chow, J. Mallet-Paret and J. A. Yorke, Finding zeroes of maps: homotopy methods that are constructive with probability one (preprint).

2. S. Friedland, Matrices with prescribed off-diagonal elements, Israel J. Math. 11 (1972), 184-189.

3. On inverse multiplicative eigenvalue problems for matrices, Linear Algebra and Appl. 12 (1975), 127-137.

4. Inverse eigenvalue problems (preprint).

5. R. B. Kellogg, T. Y. Li and J. A. Yorke, A method of continuation for calculating a Brouwer fixed point, Fixed Points, Algorithms and Applications, S. Karamadian (editor), Academic Press, New York, 1977.

6. A constructive proof of the Brouwer Fixed Point Theorem and computational results, SIAM J. Numer. Anal. 13 (1976), 473-483.

7. J. W. Milnor, Topology from the differential viewpoint, Univ. of Virginia Press, Charlottesville, Va., 1965.

Department of Mathematics, University of Maryland, College Park, Maryland 20742

Current address (until September 1978): Mathematisches Institut der Universität Bonn, 5300 Bonn 1, Federal Republic of Germany 